\section{Transtorno do déficit de atenção e hiperatividade (TDAH): aspectos relacionados à comorbidade com distúrbios da atividade motora}

\section{Attention-deficit hyperactivity disorder: aspects related to motor activity co- morbidity}

Heloisa S. Pereira 1

Alexandra P. Q. C. Araújo 2

Paulo Mattos 3

1 Departamento Materno-Infantil. Disciplina de Pediatria. Faculdade de Ciências Médicas. Universidade Estadual do Rio de Janeiro. Av. Prof. Manoel de Abreu, 444, $2^{\circ}$. andar. Vila Isabel. Rio de Janeiro, RJ, Brasil. CEP: 20.550-170 E-mail: heloisap@uerj.br

2 Departamento de Neuropediatria. Universidade Federal do Rio de Janeiro. Rio de Janeiro, RJ, Brasil.

3 Departamento de Psiquiatria. Universidade Federal do Rio de Janeiro, RJ. Brasil.

\begin{abstract}
This paper addresses the physiopathological and clinical aspects related to Attention-Deficit Hyperactivity Disorder in particular the ones concerning the association of this disorder with the Coordination Development Disorder. The Medline database was used to survey indexed articles from 1965 thru 2004. As a result of the articles selected, others were obtained through the relevance assigned to them in the initial sources. Hereditary predisposal of this condition is unquestionable as well as the presence of noradrenergic and dopaminergic dysfunction in the pré-frontal cortex and connections. Notwithstanding this condition diagnosis is based on clinical data. Morbid associations occur in approximately half of the individuals and the prevailing comorbidities as of psychiatric nature. The presence of co-morbidity may modify the therapy and prognosis. The Coordination Development Condition, also stemming from clinical diagnosis is cause for a worse prognosis to children sharing the same conditions. An active search of associated conditions should be performed in each of the children diagnosed.
\end{abstract}

Key words Attention deficit disorder with hyperactivity, Child development, Prognosis

\section{Resumo}

A presente revisão aborda aspectos fisiopatológicos e clínicos referentes ao Transtorno do Déficit de Atenção com Hiperatividade (TDAH), em especial aqueles que concernem à associação desse transtorno com o Distúrbio do Desenvolvimento da Coordenação (DDC). Utilizou-se a base de dados Medline para levantamento de artigos indexados a partir de 1965 até 2004. Aos artigos selecionados dessa forma, outros foram obtidos pela relevância atribuída a eles nas fontes iniciais. A pré-disposição hereditária desse transtorno é indiscutivel, bem como a presença da disfunção noradrenégica e dopaminérgica no córtex pré-frontal e suas conexões. Apesar desse conhecimento, o diagnóstico da condição se baseia em dados clínicos. As associações mórbidas ocorrem em cerca de metade dos indivíduos, sendo as principais comorbidades de natureza psiquiátrica. A presença de uma comorbidade pode modificar a terapêutica e o prognóstico. O Distúrbio do Desenvolvimento da Coordenação, condição também de diagnostico clínico, confere pior prognóstico às crianças que partilham ambos os quadros. Recomenda-se que uma busca ativa de condições associadas seja realizada em cada criança diagnosticada como portadora de TDAH.

Palavras-chave Desenvolvimento infantil, Transtorno da falta de atenção com hiperatividade, Prognóstico 


\section{Introdução}

A Síndrome reconhecida atualmente como Transtorno do Déficit de Atenção e Hiperatividade (TDAH) é uma das possibilidades diagnósticas quando o profissional encontra-se diante de queixas referentes ao comportamento discrepante daquele esperado para a faixa etária e inteligência, e que acarrete prejuízo para o desenvolvimento da criança em diferentes domínios da integração social. De acordo com o Manual de Diagnóstico e Estatística nas Doenças mentais IV (Diagnostic and Statistical Manual of Mental Disorders - DSM IV) ${ }^{1}$ o diagnóstico é obtido quando o paciente atende a pelo menos seis dos nove critérios de um ou de ambos os domínios da síndrome (hiperatividade/impulsividade e desatenção) em pelo menos dois locais de avaliação distintos, como por exemplo em casa e na escola. Confere-se assim a classificação de tipo predominantemente Hiperativo/impulsivo (apenas presentes seis ou mais dos critérios de impulsividade/hiperatividade), de tipo predominatemente "Desatento" (apenas presentes seis ou mais dos critérios de desatenção), ou do tipo "Combinado". (Quadro 1)
As crianças hoje reconhecidas como portadoras de TDAH já foram denominadas sob diversas alcunhas: Déficit do Controle Moral, Síndrome da Inquietude, Lesão Cerebral Mínima, Reação Hipercinética da Infância, Doença do Déficit de Atenção com e sem Hiperatividade. As teorias desenvolvidas sobre a etiologia da TDAH refletem tendências científicas e sociais de suas épocas respectivas e que, progressivamente, foram abandonando as noções de falha disciplinar como principal efeito causal. A associação entre surtos de encefalite letárgica e sequielas comportamentais em 1922, fez com que uma base anatômica fosse proposta para o transtorno. Os avanços obtidos pelo melhor entendimento de processos neuro-químicos, estudos genéticos e o avanço na confiabilidade de estudos populacionais vêm somar-se ao corpo de saber sobre o que hoje reconhecemos como TDAH. Associações freqüentes de TDAH com transtornos habitualmente avaliados por profissionais de diversas áreas da atenção à saúde, tais como fonoaudiólogos, psicólogos e terapeutas ocupacionais, são verificadas. Dessa forma, atualmente é reconhecida e proposta a necessidade da abordagem multidisciplinar na avaliação clínica e na criação de modelos adequados de

\section{Quadro 1}

Critérios do "Diagnostic and Statistical Manual of Mental Disorders" (DSM) IV1 para Transtorno do Deficit de Atenção e Hiperatividade (TDAH), 1994.

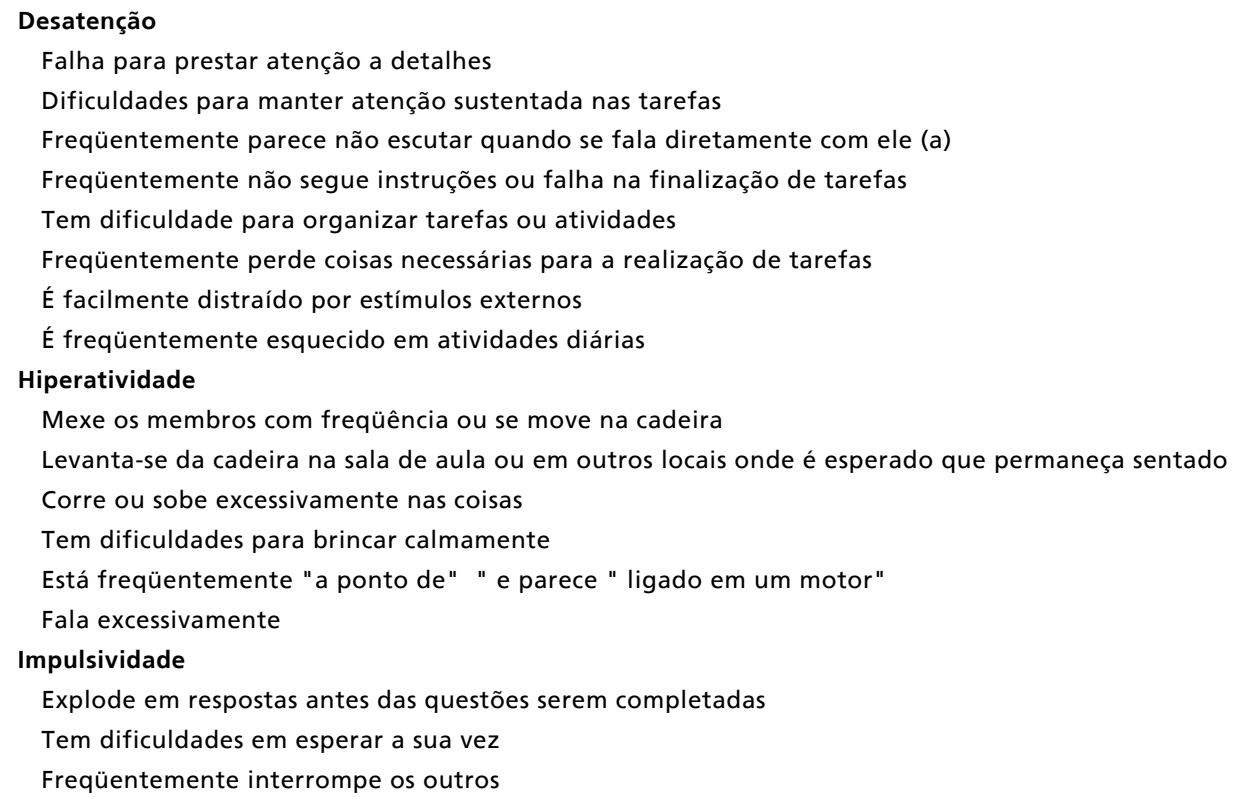


diagnóstico e intervenção para estas crianças.

Geralmente, o diagnóstico se faz na faixa etária escolar. Nesta época os sintomas de hiperatividade e desatenção freqüentemente impedem que a criança se mantenha em condições favoráveis ao aprendizado, gerando problemas secundários antes não perceptíveis ou pouco valorizados. Pré-escolares, no entanto, podem apresentar sintomas suspeitos de TDAH e alguns podem obter tal diagnóstico, mesmo que precocemente. ${ }^{2}$ Salienta-se, porém, que embora dados epidemiológicos sugiram que aproximadamente $2 \%$ dos pré-escolares possam ser diagnosticados com TDAH, ${ }^{3}$ um total de até $50 \%$ dos pais que apresentam preocupações com seus filhos pré-escolares nesse sentido, deixam de fazê-lo por época da alfabetização. ${ }^{4}$ Deve-se ter em mente que a gravidade inicial dos parâmetros de diagnóstico relacionam-se com a presença de problemas sociais e acadêmicos na adolescência.5 Por outro lado, aqueles cujas mães têm boa escolaridade, cuja família é mais estável e que têm mais fluência verbal terão prognóstico mais favorável. 6 No pré-escolar a atenção e o controle sobre a atividade motora encontram-se ainda em desenvolvimento e determinados comportamentos nesta faixa etária devem ser avaliados na perspectiva de uma fase incompleta de maturação. Ainda, múltiplas etiologias podem levar ao comportamento clínico semelhante e o diagnóstico diferencial deverá incluir alterações de base neurológica tais como exposição fetal ao álcool e complicadores perinatais, bem como minuciosa anamnese de sistemas.

O TDAH possui uma forte predisposição hereditária, ${ }^{7}$ com fator de hereditariedade que pode alcançar 0.80 .8 A prevalência varia entre 5 e $15 \%$ das crianças em idade escolar e a incidência é três vezes maior no sexo masculino. ${ }^{9}$ Alguns trabalhos identificaram a existência de famílias pré-dispostas a diversos diagnósticos psiquiátricos, incluindo TDAH. Nessas, a estimativa para que haja hereditariedade do subtipo diagnóstico (hiperativo/impulsivo, desatento ou combinado) é em torno de $60 \%$.1012 Adicionalmente, as crianças cujos sintomas de TDAH não remitem com a idade tendem a apresentar familiares afetados com mais freqüência, sugerindo um modo familiar de características mais penetrantes. 13

Alterações nos genes transportador (DAT) e receptor de dopamina (DRD4) estão freqüentemente implicadas na susceptibilidade ao TDAH, embora resultados negativos também sejam relatados, sugerindo ser a TDAH condição heterogênea de etiologia multifatorial genética e não-genética. Neste aspecto, a presença de Transtorno de Conduta e doença bipolar, comorbidades freqüentes no TDAH, e a definição de subgrupos em função das mesmas parece estratificar os pacientes em famílias homogêneas no que diz respeito às características gênicas. ${ }^{8}$ Hipoteticamente, esse modelo de subclassificação pode prever resposta diferenciada à medicações de acordo com o perfil genético. 8,14

Polimorfismos do receptor DRD4, parte do complexo de transmissão noradrenérgica, e sintomas de TDAH correlacionam-se, especialmente em adultos. A existência de disfunções noradrenérgicas no córtex pré-frontal (CPF), sugerida também por estudos de imagem funcional, 15 configura a base de uma das teorias mais convincentes para a fisiopatologia da TDAH: a disfunção neurotransmissora do CPF, determinada, em alguns casos, de forma genética. Notadamente, uma série de fatores etiológicos pode ser responsável pelo desequilíbrio funcional do córtex pré-frontal e a melhora de função noradrenérgica pode auxiliar na superação dos sintomas de forma independente da causa. 16-18

A depleção global ou parcial de catecolaminas no CPF prejudica as funções de atenção e de memória operacional, que permitem o desenvolvimento de estratégias de ação bem sucedidas. As funções mentais específicas que permitem o autocontrole são unificadas sob o termo "funções executivas". São incluídas nessa categoria as atividades volitivas, interpretativas e que suprimem informações supérfluas e distratoras. 19,20

A ação terapêutica de agentes noradrenérgicos se faz pela melhora das funções do córtex pré-frontal memória de trabalho, resposta inibitória e planejamento sob condições distratoras. 18 Ainda, evidências sugerem que os agonistas alfa-2 agem diretamente no CPF aumentando a memória de trabalho.21,22 Um modelo plausível é que essas medicações aumentem a atividade frontal inibitória através de vias dopa e noraminérgicas sobre estruturas subcorticais atingidas por desequilíbrios neurotransmissores de etiologias diversas. ${ }^{23}$ Kroes et al., ${ }^{24}$ sugerem que baixos níveis de dopamina poderiam associar-se à regulação deficitária dos sinais oriundos dos gânglios da base, justificando seus achados de associação entre alterações qualitativas da movimentação de crianças entre cinco e seis anos e o diagnóstico posterior de TDAH. A função serotoninérgica, agente indireto na modulação do sistema noradrenérgico também se encontra alterada com transtornos comportamentais. ${ }^{25-28}$

A heterogeneidade clínica da TDAH foi relacionada aos problemas no funcionamento das diversas funções executivas. Os subtipos de TDAH foram avaliados com a utilização de testes especí- 
ficos para funções executivas em 108 crianças. Os pesquisadores encontraram problemas de planejamento nos indivíduos com o subtipo combinado e marcante redução da inibição motora em meninos de ambos os subtipos. ${ }^{29}$ Em um estudo que se propôs a treinar habilidades de memória de trabalho, sete crianças com TDAH e sete controles foram treinadas intensivamente ao longo de seis semanas para a execução de tarefas relativas à memória de trabalho em jogo de computador. Um programa alternativo foi utilizado como placebo. Segundo os autores, houve melhora no desempenho de funções relativas à memória de trabalho e ao lobo frontal, sugerindo possibilidade de intervenção por treinamento que beneficie essas crianças em algum momento adiante, após a realização de trabalhos com amostras mais significativas. 30 No entanto, a eficácia do tratamento medicamentoso é comprovadamente superior à terapia comportamental. 31

Estudos reconheceram a existência de TDAH em diferentes culturas,32,33 sendo as taxas de prevalência aproximadas quando se utilizam critérios semelhantes. É sabido também, que fatores culturais e sociais modulam a percepção clínica de transtornos comportamentais 32,34 o que, associado a questões relativas à metodologia adotada, tais como instrumentos utilizados e critérios de seleção de amostra, explicam diferentes prevalências entre culturas, podendo chegar a $17 \%$ na Alemanha 35 enquanto que, nos EUA, encontra-se em torno de 5\%.6

A ausência de marcadores biológicos utilizáveis na prática clínica faz com que o diagnóstico se embase na anamnese, analisada em conjunto com fatores tais como aspectos psicossociais e peculiaridades de cada população. Rohde et al.,36 demonstraram a validade de critérios para a população brasileira do Rio Grande do Sul e concluíram que, guardadas as considerações de familiaridade do entrevistador com o esperado para a população, é possível utilizar, de maneira confiável, os critérios do DSM IV no Brasil. Os resultados de estudos populacionais sobre TDAH apontam para uma condição de apresentação clínica variada e representativa de um continuum de alterações de atenção e hiperatividade/impulsividade. 37

\section{Comorbidades e sua importância}

A presença de comorbidades é uma situação extremamente comum em indivíduos com TDAH e deve ser ativamente checada durante a fase diagnóstica. 38 A associação de TDAH com outras condições clínicas encontra-se entre 30 e $50 \%$ dos casos, embora em algumas situações esse número possa ser ainda maior. ${ }^{6}$ A alta freqüência presença de associações mórbidas sugere a existência de um traço contínuo, manifesto de forma variável por estes indivíduos e que, através de base fisiopatológica comum possa também resultar na manifestação isolada das condições ora co-ocorrentes.

As condições associadas abrangem aspectos da aquisição e execução da comunicação falada e escrita, transtornos de aprendizado, transtornos do humor e da personalidade e transtorno de uso de substâncias. Ainda, a capacidade de adquirir apropriadamente aptidões motoras finas e grosseiras, também chamado de "distúrbio do desenvolvimento da coordenação", é uma associação freqüente. Hechtman et al.39 avaliaram o prognóstico da TDAH associada a outras condições e propuseram a existência de três categorias de prognóstico estratificadas em gravidade. O primeiro grupo funcionaria muito bem na vida adulta e deteria $30 \%$ das crianças. Um segundo grupo continuaria tendo problemas de impulsividade, concentração e inserção social, comprazendo 50 a $60 \%$ das crianças. Um terceiro grupo, contendo 10 a $15 \%$ dos indivíduos teria sérios problemas psiquiátricos e sociais, depressão, uso de drogas e álcool, ou conduta anti-social.

Dentre os fatores que influenciam o diagnóstico de TDAH encontra-se a capacidade cognitiva global.40 O retardo mental não é um comemorativo esperado do TDAH, embora possuam alguns fatores etiológicos em comum. O QI é preditor específico do rendimento acadêmico, conferindo pior prognóstico quando associado ao TDAH. Mesmo em crianças que têm QI na faixa normal, a competência acadêmica e prognóstico final encontram-se relacionados à estratificação do QI,40 ocorrendo a necessidade de inclusão de crianças em programas de suporte acadêmico relacionada com problemas emocionais na adolescência. ${ }^{41}$ Também na resposta ao tratamento, faz-se notar a importância do QI, Handen et al.42 demonstraram que crianças com TDAH e retardo mental leve mostraram melhora clínica mais acentuada com o uso de medicação estimulante do que aqueles com TDAH e graus maiores de retardo mental.

Outro fator que influencia o prognóstico final é o perfil de apresentação de TDAH. Hechtman et al.43 mostraram que a hiperatividade inicial, em conjunto com outros fatores sociais e familiares prediziam o ajustamento social, o desempenho escolar os registros de trabalho e o número de acidentes de carro. A impulsividade, hiperatividade e atitudes potencialmente anti-sociais foram preditivos do diagnóstico de transtorno opositivo desafiador 44 e transtorno de 
conduta precoce. 45 É estimado que 40 a $60 \%$ das crianças com TDAH tenham comportamento opositivo desafiador (TOD) como comorbidade. Barkley et al.46,47 demostraram que os indivíduos com TDAH que também apresentavam TOD tinham relações mais negativas com suas mães, mais conflitos domésticos e maior estresse psicológico materno. A comorbidade com TOD torna-se importante também por ser preditiva de Transtorno de Conduta (TC), condição mais séria e de conseqüências mais dramáticas. A presença de Transtorno de Conduta piora o prognóstico do TDAH 48 com maior prevalência de uso de tabaco e álcool e maior número de suspensões e expulsões escolares e delinqüência. Em famílias de crianças que apresentam TDAH e Transtorno de Conduta ocorre maior prevalência de pais que utilizam drogas de maneira abusiva, depressão ou transtornos de personalidade anti-social. Parece haver uma agregação biológica não casual entre TDAH e distúrbios de conduta. 49 Faraone, 8 em estudo de meta-análise, sugere transmissão associada em famílias de TDAH, distúrbios de conduta e doença bipolar.

Os distúrbios do humor, depressão e ansiedade estão presentes em 20 a $40 \%$ dos indivíduos com TDAH,50,51 mas seu impacto no prognóstico é menos negativo que aquele da associação com Transtorno de Conduta.52 A presença de agressividade, por sua vez, é altamente preditiva de comportamento anti-social na adolescência e de violência na vida adulta. $53 \mathrm{Em}$ estudo realizado por Souza et al.,54 o perfil de comorbidades foi avaliado em 34 crianças e adolescentes. Nessa amostra $85,7 \%$ das crianças apresentava comorbidades, sendo que $20,6 \%$ apresentavam TOD, 39,2\% apresentavam TC e $57 \%$ das crianças apresentavam mais de um diagnóstico de comorbidade incluindo a combinação entre os já citados, depressão e estados ansiosos diversos.

O desempenho acadêmico pode ser afetado pelos sintomas de TDAH e/ou de suas comorbidades. Dentre essas, encontram-se transtornos específicos do aprendizado tais como a dislexia. Problemas de atenção parecem relacionados com problemas acadêmicos mais tardios. A relação entre atenção e desempenho acadêmico foi linear, com grave desatenção inicial relacionada ao pior prognóstico escolar. ${ }^{55}$ Em crianças com TDAH, a prevalência de transtornos de aprendizado fica entre $20 \%$ e $80 \%$, sendo que as grandes variações ficam por conta de diferenças metodológicas. ${ }^{56,57} \mathrm{~A}$ pesquisa na área sugere que os problemas de atenção e aqueles de aprendizado sejam entidades separadas, mas freqüentemente co-ocorrentes.58,59 A falência acadê- mica de uma criança com TDAH, portanto, pode dever-se à co-ocorrência de transtornos de aprendizado. Das crianças com tal diagnóstico, $80 \%$ têm problemas de leitura. 60 Os déficits convergem para problemas relativos ao processamento fonológico. 61,62 Algumas crianças apresentam transtornos de aprendizado não verbal, nos quais a forma e o conteúdo da linguagem falada estão preservados, mas a aritmética está deficiente. Essas crianças freqüentemente têm problemas com aptidões espaciais de organização e sociais. Os problemas de matemática são particularmente comuns, mas a presença de Trasntorno da Aritmética é rara, uma vez que os déficits observados são primariamente devidos à desatenção. 63

Por outro lado, aqueles que recebem primariamente o diagnóstico de transtorno de aprendizado devem ser avaliados para TDAH, já que, a incidência de TDAH nesse grupo varia entre $9 \%$ e $92 \%$, dependendo da amostra populacional avaliada.64 Dentre as influências de baixo desempenho acadêmico na vida adulta encontram-se relatadas a diminuição do número de anos de escolaridade sem necessariamente afetar as aptidões de leitura e a eventual persistência dos problemas de leitura. 19 Naqueles com transtornos de aprendizado, encontra-se correlação positiva na vida adulta com comportamento delinqüente, baixa auto-estima, mais impulsividade, inquietação, imaturidade e desatenção. Assim, podemos considerar a importância da avaliação de aptidões que sugiram esta condição de grandes conseqüências.

\section{Distúrbio do desenvolvimento da coordenação}

Um grupo de comorbidades para a qual se devota especial atenção no presente artigo é aquela encontrada entre TDAH e problemas motores, também designadas como Distúrbio do Desenvolvimento da coordenação (DDC). ${ }^{1}$ As crianças com TDAH foram há muito reconhecidas como tendo maior quantidade e diferente qualidade de movimento, quando comparados com controles. ${ }^{65}$ Flutuações observadas na atividade motora sugeriram, ainda nos anos 70 , que houvesse falha na regulação da atividade motora para atender às demandas motoras específicas de cada situação. 66 Esses achados foram mais recentemente avaliados e serão abordados mais adiante. Atualmente se aceita a combinação entre TDAH e DDC como um evento comum, que pode chegar a $50 \%$ das crianças com TDAH, 6 sendo que na população geral a prevalência de DDC isolada é estimada 
Critérios para o diagnóstico de disfunção motora conforme os critérios do "Diagnostic and Statistical Manual of Mental Disorders" (DSM) IV1IV e do "Classificação Internacional de Doenças (CID)" 1069.

\begin{tabular}{|ll|}
\hline $\begin{array}{l}\text { DSM IV } \\
\text { Distúrbio do Desenvolvimento da Coordenação }\end{array}$ & $\begin{array}{l}\text { CID 10 } \\
\text { Distúrbio específico do desenvolvimento da função } \\
\text { motora }\end{array}$ \\
\hline $\begin{array}{l}\text { Atividades de vida diária depen-dentes de coordenação } \\
\text { motora encontram-se consideravelmente abaixo do } \\
\text { esperado em função da idade cro-nológica e do } \\
\text { coeficiente cognitivo. }\end{array}$ & $\begin{array}{l}\text { Ootora grosseira ou fina encontra-se pelo menos a } 2 \\
\text { cronológica da criança. }\end{array}$ \\
$\begin{array}{l}\text { Tal defasagem interfere no desem-penho acadêmico ou } \\
\text { no dia-a-dia. }\end{array}$ & $\begin{array}{l}\text { O distúrbio interfere de forma significante no no } \\
\text { desempenho acadêmico ou nas atividades de vida diária. }\end{array}$ \\
$\begin{array}{l}\text { Não relacionado à outra condição (como paralisia } \\
\text { cerebral, transtorno invasivo do desenvolvimento...) }\end{array}$ & Não há outro diagnóstico neuro-lógico. \\
$\begin{array}{l}\text { Caso haja retardo mental as dificuldades motoras devem } \\
\text { estar aquém das cognitivas. }\end{array}$ & $\begin{array}{l}\text { Ficam excluídas crianças com coe-ficiente de inteligência } \\
\text { abaixo de } 70 \text { em teste estandartizado. }\end{array}$ \\
\hline
\end{tabular}

em torno de $10 \%$. A presença de complicações perinatais se nota mais freqüentemente nessas crianças quando comparadas com controles. $67,68 \mathrm{De}$ acordo com o DSM IV:

"... Indivíduos com TDAH podem cair, esbarrar em coisas ou derrubar objetos mas isto é geralmente devido à distração e impulsividade, mais do que por deficiência motora. Se o critério para as duas doenças (TDAH e DDC) é preenchido então os diagnósticos estão associados. "1. No CID-1069, o transtorno recebe o nome de "distúrbio específico do desenvolvimento da função motora." Ambas as descrições encontram-se expressas na Quadro 2.

Acredita-se que a principal causa do DDC seja uma incapacidade no planejamento e execução de ações motoras - dispraxia do desenvolvimento. ${ }^{70-73}$ A dispraxia do desenvolvimento é caracterizada por julgamentos inadequados de seqüência, tempo e força e reflete uma alteração cuja localização anatômica ainda não é precisa. Quando utilizamos ambas as mãos na execução de movimentos finos necessitamos das conexões inter-hemisféricas ${ }^{74}$. Sincronia alterada dos movimentos pode ser observada em indivíduos com lesão de lobo parietal, prejudicando a coordenação manual. 75 Finalmente, estudos com animais de laboratório implicam o sistema dopaminérgico nigro-estriatal. 76 Evidentemente, outros processos cognitivos, sensoriais e motores estão envolvidos na dificuldade do planeja- mento e execução de atos motores, incluindo propriocepção e visão.67,73,77-79 A concomitância entre os diagnósticos de DDC e TDAH foi observada e, descrita em países escandinavos durante a década de 80 e originou o termo descritivo DAMP (Deficits na Atenção, Controle Motor e Percepção), utilizado para descrever a combinação clínica das duas entidades. $80 \mathrm{O}$ diagnóstico é feito através de cuidadoso exame motor associado à positividade de critérios para TDAH (Quadro 3).

Vários testes foram propostos para avaliar o distúrbio da coordenação.80-85 Apesar disso, não há recomendação específica para o uso de qualquer deles no diagnóstico, estando a escolha a critério do usuário.

Crianças com DDC têm dificuldades com habilidades motoras que requerem coordenação, além de dificuldades no desempenho acadêmico e de sociabilização. Tarefas quotidianas simples como amarrar os sapatos ou abotoar a camisa podem ser especialmente difíceis. As crianças têm QI geralmente dentro dos limites da normalidade, mas podem apresentar dificuldades em áreas não-motoras, como, por exemplo, na linguagem. ${ }^{1}$ As ferramentas diagnósticas aferem os processos responsáveis pela atividade motora fina e coordenada. Problemas na função integrativa resultam em movimentos mal planejados, estereotipados e mal-sucedidos, quando a criança interage com o ambiente. 86 De acordo com Laszlo et al., 87 problemas motores podem ser melhorados de 
Critérios propostos para o diagnóstico de DAMP (Déficits de Atenção, controle Motor e Percepção). Gillberg C et al.80

\begin{tabular}{|c|c|}
\hline & \\
\hline Pular em uma perna 20 vezes & $\begin{array}{l}\text { Francamente anormal se levar mais que } 12 \text { segundos ou } \\
\text { interromper por duas vezes com ambos os pés no chão; }\end{array}$ \\
\hline $\begin{array}{l}\text { Ficar em pé em uma perna por } 20 \\
\text { segundos }\end{array}$ & Consegue por $<\mathrm{ou}=10$ segundos; \\
\hline $\begin{array}{l}\text { Andar na face lateral dos pés por } 10 \\
\text { segundos }\end{array}$ & $\begin{array}{l}\text { Presença de movimentos involuntários associados (Flexão } \\
\text { do cotovelo de } 60 \text { graus ou mais com abdução dos braços } \\
\text { ou movimentos de lábios e língua) }\end{array}$ \\
\hline $\begin{array}{l}\text { Movimentos alternados das mãos } \\
\text { por } 10 \text { segundos }\end{array}$ & $\begin{array}{l}\text { Consegue } 10 \text { ou menos prono-supinações para a mão não } \\
\text { dominante ou apresenta movimento errático de prono- } \\
\text { supinação com o cotovelo movendo-se mais que } 15 \mathrm{~cm} \text {; }\end{array}$ \\
\hline $\begin{array}{l}\text { Cortar um círculo de papel com } 10 \\
\mathrm{~cm} \text { de diâmetro }\end{array}$ & $\begin{array}{l}\text { 1/5 da área incluída ou excluída ou leva mais que dois } \\
\text { minutos para completar a tarefa; }\end{array}$ \\
\hline $\begin{array}{l}\text { WISC teste do labirinto de acordo } \\
\text { com o descrito no manual }\end{array}$ & $\begin{array}{l}\text { Escore }>2 \text { SD abaixo da média para a idade ( } 8 \text { ou mais dos } \\
21 \text { pontos possíveis); }\end{array}$ \\
\hline $\begin{array}{l}\text { Em pé com os braços estendidos por } \\
20 \text { seg }\end{array}$ & $\begin{array}{l}\text { Movimentos coreiformes: "mão em pá' " (flexão dos } \\
\text { punhos com extensão da articulação dos dedos) ou desvio } \\
\text { vertical ou horizontal dos braços; }\end{array}$ \\
\hline Pinça do lápis & Dois ou mais dedos opostos ao polegar; \\
\hline $\begin{array}{l}\text { Andar na ponta dos pés por } 20 \\
\text { passos }\end{array}$ & $\begin{array}{l}\text { Só consegue levantar o calcanhar por alguns passos ou } \\
\text { estende marcadamente os braços e mãos e/ou abduz o } \\
\text { antebraço e/ou faz movimentos de boca e língua; }\end{array}$ \\
\hline Motor grosseiro & $\begin{array}{l}\text { Dificuldades para pular em um pé só ou tem marcha } \\
\text { disritmica ou bate duas vezes em objetos durante o } \\
\text { exame. }\end{array}$ \\
\hline
\end{tabular}

forma consistente por programas de treinamento que focalizem as habilidades de percepção e movimento. Outros autores encontram prognóstico reservado quanto à melhora de capacidades motoras. $81,88,89$

O tempo necessário para realizar tarefas manuais objetivas foi testado em crianças com DDC e crianças controle. 90 Crianças com DDC mostraram tempos prolongados na realização de tarefas, principalmente quando o alvo era pequeno ou quando o estímulo apresentado era da variedade proprioceptiva. Os autores sugerem que esses achados são indicativos de uma redução de recursos no planejamento e controle do movimento. Outra interpretação possível é que os indivíduos lentifiquem o movimento ou diminuam seus graus de liberdade como parte de estratégia motora que lhes permita controlar a variabilidade mais acentuada de seus atos motores. ${ }^{91}$ Ainda, considerando a teoria de que essas crianças apresentam um ruído na sinalização de impulsos motores, 92 utilizariam estratégias motoras como a lentificação de movimentos, contração simultânea de agonistas e antagonistas (co-contração) e, eventualmente, a realização de movimentos balísticos de pouca relação com informações visuais na tentativa de minimizar os efeitos de informação motora inadequada. 93

Assim como nos pacientes com TDAH, encontramos uma alta prevalência de co-morbidades quando o diagnóstico primário é de DDC. Estudos de pacientes com DDC encontraram com deficits perceptivos e déficits no aprendizado da matemática, todos francamente discrepantes das medidas de 
QI.94,95 Problemas com a escrita podem revelar alterações envolvendo a coordenação, distúrbios primários de linguagem, déficits visoespaciais, problemas de atenção e memória e problemas de sequenciamento. 96 Nota-se, portanto, que não apenas a alta prevalência de comorbidades mas também as suas categorias, assemelham-se àquelas encontradas no TDAH.

Uma seqüência de estudos vem avaliando a associação entre TDAH e DDC quanto a aspectos da aptidão motora. Atrasos na aquisição de marcos do desenvolvimento foram especificamente descritos na aptidão de pedalar e no início da linguagem falada. Ainda, o mesmo estudo descreve propensão aumentada a acidentes e condicionamento físico abaixo do esperado para a idade. $97 \mathrm{O}$ alcance de menores níveis de força durante contração muscular e maiores graus de co-ativação, sugerem que haja um baixo nível de organização neuromuscular, cujas causas encontramse em investigação. 98

Estudos que avaliaram a capacidade de planejamento motor antecipado em crianças com TDAH e DDC demonstraram que existe falha em um subgrupo de indivíduos quanto ao aprendizado motor de tarefas novas, bem como no manejo de objetos corriqueiros, como copos e caixas de leite. Para esses objetos, a força aplicada pelos indivíduos se fazia de forma variável e inconstante. A variabilidade acentuada na manutenção de uma força constante mostrou-se uma característica importante desse grupo de indivíduos, podendo variar em intensidade e mesmo acomenter alguns indivíduos de outros grupos, sugerindo gradações de acometimento.99,100 Em estudo mais recente, comparando a execução de um ato motor com e sem auxílio da visão, documentou-se também maior variabilidade de respostas e piora do desempenho sem auxílio da visão, especialmente nas crianças que apresentavam TDAH e DDC, comparados com TDAH e com controles. ${ }^{101}$

Uma série de trabalhos vem examinando o prognóstico de crianças ditas "desajeitadas".88,95,102104 As conclusões sinalizam que uma boa parte das crianças não supera suas dificuldades motoras e que, a essas, somam-se prejuízos do desenvolvimento em outras áreas.

Gillberg et al. ${ }^{81,89}$ realizaram um dos primeiros estudos de seguimento de crianças com problemas motores e TDAH e examinaram pacientes com diagnóstico de DAMP aos sete anos e subseqüentemente aos 10 e 13 anos e idade. Apesar de uma grande percentagem dos pacientes diagnosticados aos sete anos ter ficado livre dos sintomas, algumas crianças com diagnósticos motor e atentivo associados mostravam problemas de escolaridade, sociabili- zação e controle emocional. Os autores sugerem que haja um programa de saúde escolar com o objetivo de identificar e tratar precocemente tais crianças. Mais recentemente estes mesmos pacientes foram revistos aos 22 anos. Os grupos de TDAH em associação com DDC e aqueles com DDC isolado obtiveram os piores desfechos, com 69 e $80 \%$, respectivamente, gravemente afetados. Algumas das crianças severamente afetadas desenvolveram manifestações autistas na vida adulta. Outras, com escores sugestivos de doença do espectro autista na infância apresentaram regressão dos sintomas ou deram início a outros problemas psiquiátricos tais como depressão maior, distúrbio de personalidade, diminuição de anos na escola e velocidade de leitura marcadamente reduzida. Esses desfechos ocorreram em 55\% das crianças com TDAH e DDC contra $11 \%$ do grupo controle. No grupo considerado gravemente afetado esse número chegou a $77 \% .103 \mathrm{Em}$ outro estudo de seguimento, 88 as crianças foram avaliadas aos oito anos e re-examinadas na adolescência. Após a primeira avaliação, seguiram um programa de treinamento durante um ano, após o qual apresentaram melhora pequena e não duradoura dos sinais motores e identificaram $65 \%$ de falha na educação física por época da adolescência, $73 \%$ com baixa auto-estima, $71 \%$ com mau rendimento escolar e $82 \%$ mostraram diagnósticos psiquiátricos relacionados à personalidade, tais como distúrbio de conduta bem como síndromes clínicas tais como ansiedade Os autores sublinham a possibilidade de um aspecto crônico da doença com prognóstico reservado quanto ao aprendizado e a sociabilização. Esses resultados são semelhantes aos de Hellgren $e t$ al., 104 na Suécia.

Polatajko et al. 105 sugerem que o treinamento repetitivo de aptidões específicas parece ser o mais útil para estas crianças. Nesse aspecto, a escola e a família devem estar orientadas a agir de modo a incentivar a realização de tarefas de modo independente, minimizando os efeitos sociais do problema, que acabam interferindo na auto-estima e auto-confiança física da criança. Essa falha no desenvolvimento de atividades físicas competitivas faz com que a criança assuma freqüentemente postura sedentária e passe a considerar-se como "não-atlética", relacionando-se com crianças que têm as mesmas dificuldades. O uso de medicamentos foi verificado como benéfico em trabalhos que avaliaram a disfunção na escrita de crianças com TDAH e dificuldades no controle motor fino. 106 Os efeitos da medicação sobre os sintomas cardinais do TDAH, parecem semelhante em indivíduos com e sem DDC associada. 97 


\section{Considerações finais}

O processo diagnóstico de um indivíduo com queixas de comportamento discrepante daquele esperado para a faixa etária e inteligência e que acarrete prejuízo para o seu desenvolvimento em diferentes domínios da integração social não se limita ao preenchimento dos critérios de TDAH pela DSM-IV ou ICD-10, uma vez que há necessidade de se excluir outros diagnósticos, tanto diferenciais,

\section{Referências}

1. American Psychiatric Association. Diagnostic and statistical manual of mental disorders. $4^{\text {th }}$ ed. Washington (DC); 1994.

2. Blackman JA. Attention-deficit hyperactivity disorder in preschoolers. Does it exist and should we treat it? Pediatr Clin North Am 1999; 46: 1011-25.

3. Lavigne JV, Gibbons RD, Christoffel KK, Arend R, Rosenbaum D, Binns H, Dawson N, Sobel H, Isaacs C. Prevalence rates and correlates of psychiatric disorders among preschool children. J Am Acad Child Adolesc Psychiatry 1996; 35: 204-14

4. Blackman JA, Levigne MD. A follow up study of preschool children evaluated for developmental and behavioral problems. Clin Pediatr 1987; 26: 248-52.

5. Pierce EL, Campbell SB, Diagnostic status and symptomatic behavior of hard to manage preschool children in middle childhood and early adolescence. J Clin Child Psychol 1999; 28: 44-57.

6. Barkley RA. Attention deficit hyperactivity disorder. a handbook for diagnosis and treatment. 2nd ed. London: Guillford; 1998.

7. Goodman R, Stevenson J. A twin study of hyperactivity 1. An examination of hyperactivity scores and categories derived from Rutter teacher and parent questionnaires. J Child Psychol Psychiatry 1989; 30: 671-89.

8. Faraone SV, Biederman J, Mennin D, Russell R Tsuang MT. Familial subtypes of attention deficit hyperactivity disorder: a four year follow-up study of children from antisocial ADHD families. J Child Psychol Psychiatry 1998; 39: 1045-53.

9. Cunninghan CE, Barkley RA. The role of academic failure in hyperactive behavior. J Learn Disabil 1978; 11: 274-80.

10. Hudziak JJ, Heath AC, Madden PF, Reich W, Bucholz KK, Slutske W, Bierut LJ, Neuman RJ, Todd RD. Latent class and factor analysis of DSM-IV ADHD: a twin study of female adolescents. J Am Acad Child Adolesc Psychiatry 1998; 37: 848-57.

11. Levy E, Hay DA, McStephen M, Wood C, Waldman I. Attention-deficit hyperactivity disorder: a category or a continuum? Genetic analysis of a large scale twin study. J Am Acad Child Adolesc Psychiatry 1997; 36: 737-44. quanto modificadores do prognóstico e tratamento. Para as crianças com TDAH, uma avaliação sistemática de comorbidades auxilia a correta orientação da família quanto aos problemas que podem, inclusive, ser mais prejudiciais que o próprio TDAH ou, somar-se a ele, agravando o prognóstico final. Naqueles que apresentam o diagnóstico associado de DDC, especial atenção deve ser dedicada à identificação de indicadores de prejuízo acadêmico e pobre adaptação social, dedicando-se maiores esforços na promoção de atividades que visem minorar um pior prognóstico.

12. Sherman DK, Iacono WG, McGue MK. Attention-deficit hyperactivity disorder dimensions: a twin study of inattention and impulsivity-hyperactivity. J Am Acad Child Adolesc Psychiatry 1997; 36: 745-53.

13. Faraone SV. Genetics of childhood disorders: XX. ADHD, Part 4: is ADHD genetically heterogeneous? J Am Acad Child Adolesc Psychiatry 2000; 39: 1455-7.

14. Todd RD. Genetics of childhood disorders: XXI. ADHD, part 5: a behavioral genetic perspective. J Am Acad Child Adolesc Psychiatry. 2000; 39: 1571-3.

15. Ernst M, Zametkin AJ, Matochik JA, Jons PH, Cohen RM. DOPA decarboxylase activity in attention deficit disorder adults: a (fluorine-18) fluorodopa positron emission tomographic study. J Neurosci 1998; 18: 5901-7.

16. Arnsten AFT. Genetics of childhood disorders: XVIII. ADHD, Part. 2: norepinephrine has a critical modulatory influence on prefrontal cortical function. J Am Acad Child Adolesc Psychiatry 2000; 39: 1201-3.

17. Arnsten AFT. Catecholamine modulation of prefrontal cortical cognitive function. Trends Cogn Sci 1998; 11: 43647.

18. Jakala P, Riekkinen M, Sirvio J, Koivisto E, Kejonen K, Vanhanen M, Riekkinen P Jr. Guanfacine, but not clonidine, improves planning and working memory performance in humans. Neuropsychopharmacology 1999; 20: 460-70

19. Barkley RA. Sense of time in children with ADHD, effects of duration, distraction and stimulant medication. J Int Neuropsychol Soc 1997; 3: 359-69.

20. Lezak MD. Neuropsychological assessment. New York: Oxford University Press; 1995.

21. Avery R, Franowicz JCS, Studholme C, van Dyck CH, Arnsten AFT. The alpha-2A-adrenoceptor agonist, guanfacine, increases regional cerebral blood flow in dorsolateral prefrontal cortex of monkeys performing a spatial working memory task. Neuropsychopharmacology 2000; 23: $240-9$

22. Biederman J, Spencer TJ. Genetics of childhood disorders: XIX. ADHD. Part 3: Is ADHD a noradrenergic disorder? J Am Acad Child Adolesc Psychiatry 2000; 39: 1330-3. 
23. Zametkin AJ, Rapoport JL. Noradrenergic hypothesis of attention deficit disorder with hyperactivity: a critical review. In: Meltzer HY, editor. Psychopharmacology: the third generation of progress. New York: Raven, 1987. p. 837-42.

24. Kroes M, Kessels AG, Kalff AC, Feron FJ, Vissers YL, Jolles J, Vles JS. Quality of movement as a predictor of ADHD: results from a prospective population study of 5 and 6 year old children. Dev Med Child Neurol 2002; 44 : 753-60.

25. Kruesi MJ, Rapoport JL, Hamburger S, Hibbs E, Potter WZ, Lenane M. Brown GL. Cerebrospinal fluid monoamine metabolites, aggression and impulsivity in disruptive behavior disorders of children and adolescents. Arch Gen Psychiatry 1990; 47: 419-26.

26. Quist FJ, James LBS, Kennedy MD. Genetics of childhood disorders: XXIII. ADHD, Part 7: the serotonin system. J Am Acad Child Adolesc Psychiatry 2001; 40: 253-6.

27. Pine DS, Coplan JD, Wasserman GA, Miller LS, Fried JE, Davies M, Cooper TB, Greenhill L, Shaffer D, Parsons B. Neuroendocrine responses to fenfluramine challenge in boys. associations with aggressive behavior and adverse rearing. Arch Gen Psychiatry 1997; 54: 839-46. Erratum in: Arch Gen Psychiatry 1998; 55: 625.

28. Spivak B, Vered Y, Yoran-Hegesh R, Averbuch E, Mester R, Graf E, Weizmen A. Circulatory levels of catecolamines, serotonin and lipids in attention deficit hyperacivity disorder. Acta Psychiatr Scand 1999; 99: 300-4.

29. Nigg JT, Blaskey LG, Huang-Pollock CL, Rappley MD. Neuropsychological executive functions and DSM IV ADHD subtypes. J Am Acad Child Adolesc Psychiatry 2002; 41: 59-66.

30. Klingberg T, Forssberg H, Westerberg H. Training of working memory in children with ADHD. J Clin Exp Neuropsychol 2002; 24: 781-91.

31. MTA Cooperative Group. A 14-month randomized clinical trial of treatment strategies for attention deficit/hyperactivity disorder. Multimodal Treatment study of Children with ADHD. Arch Gen Psychiatry 1999; 56: 1073-86.

32. Livingston R. Cultural issues in diagnosis and treatment of ADHD. J Am Acad Child Adolesc Psychiatry 1999; 38 : 1591-4.

33. Rohde LA, Biederman J, Busnello EA, Zimmermann H, Schmitz M, Martins S, Tramontina S. ADHD in a school sample of Brazilian adolescents: a study of prevalence, comorbid conditions and impairments. J Am Acad Adolesc Psychiatry 1999; 38: 716-22.

34. Reid AH, Ballinger BR.. Behavior symptoms < among severely and profoundly mentally retarded patients. A 1618 years follow up study. Br J Psychiatry 1995; 167: 452-5.

35. Baumgaertel A, Wolraich ML, Dietrich M. Comparison of diagnostic criteria for attention deficit disorders in a german elementary school sample. J Am Acad Child Adolesc Psychiatry 1995; 34: 629-38.

36. Rohde LA. ADHD in Brazil: the DSM IV criteria in a culturally different population. J Am Acad Child Adolesc Psychiatry 2002; 41: 1131-3.

37. Levy F, Hobbes G. Discrimination of attention deficit hyperactivity disorder by the continuous performance test. J Paediatr Child Health 1997; 33: 384-7.
38. Spencer T, Biederman J, Wilens T. Attention deficit/hyperactivity disorder and comorbidity. Pediatr Clin North Am 1999; 46: 915-27.

39. Hechtman L. Predictors of long term outcome in children with attention deficit/hyperactivity disorder. Pediatr Clin North Am 1999; 46: 1039-52.

40. Weiss G, Minde K, Werry JS, Douglas V, Nemeth E. A five years follow up study of 91 hyperactive school children. Arch Gen Psychiatry 1971; 24: 409-14.

41. Feehan M, McGee R, Williams SM, Nada-Raja S. Models of adolescent psychopathology: childhood risk and the transition to adulthood. J Am Acad Child Adolesc Psychiatry 1995; 34: 670-9.

42. Handen BL, Janosky J, McAuliffe S. Long-term follow-up of children with mental retardation/borderline intellectual functioning and ADHD. J Abnorm Child Psychol 1997; 25 : 287-95.

43. Hechtman L, Weiss G, Perlman T, Amsel R. Hyperactives as young adults: initial predictors of adult outcome. J Am Acad Child Adolesc Psychiatry 1984; 25: 250-60.

44. Fischer M, Barkley RA, Fletcher KE, Smallish L. The adolescent outcome of hyperactive children: predictors of psychiatric, academic, social and emotional adjustment. J Am Acad Child Adolesc Psychiatry 1993; 34: 499-509.

45. Loeber R, Green SM, Keenan K, Lahey BB. Wich boys will fare worse? Early predictors of the onset of conduct disorder in a six year longitudinal study. J Am Acad Child Adolesc Psychiatry 1995; 34: 499-509.

46. Barkley RA, Fischer M, Edelbrock C, Smallish L. The adolescent outcome of hyperactive children diagnosed by research criteria: an eight years follow up study. J Am Acad Child Adolesc Psychiatry 1990; 29:546-57.

47. Barkley RA, Fischer M, Edelbrock C, Smallish L. The adolescent outcome of hyperactive children diagnosed by research criteria. III. Mother-child interactions, family conflicts and maternal psychopathology. J Child Psychol Psychiatry 1991; 32: 233-55.

48. August GJ, Steward MA, Holmes CS. A four year follow up of hyperactive boys with and without conduct disorder. Br J Psychiatry 1983; 143: 192-8.

49. Szatamari P, Boyle MH, Offord DR. Familial aggregation of emotional and behavioral problems of childhood in the general population. Am J Psychiatry 1993; 150: 1398-403.

50. Biederman J, Faraone S, Lapey K. Comorbidity of diagnosis in attention deficit hyperactivity disorder. Child Adolesc Psychiatric Clin North Am 1992; 1: 335-60.

51. Bird HR, Gould MS, Staghezza BM. Paterns of diagnostic comorbidity in a community sample of children aged nine through 16 years. J Am Acad Child Adolesc Psychiatry 1993; 32: 361-8.

52. Harrington R, Foudge H, Rutter M, Pickles A, Hill J. Adult outcome of childhood and adolescent depression. II: Links with antisocial disorder. J Am Acad Child Adolesc Psychiatry 1991; 30: 434-9.

53. Akerman P, Dykman R, Peters J, Teenage status of hypearactive and non-hyperactive learningdisabled boys. Am J Orthopsychiatry 1977; 47: 577-96.

54. Souza I, Serra MA, Mattos P, Franco VA. Comorbidity in children and adolescents with attention deficit disorder: preliminary results. Arq Neuropsychiatry 2001; 59 (2B): 401-6. 
55. Fergusson DM, Linskey MT, Horwood LJ. Attentional difficulties in middle childhood and psychological outcomes in young adulthood. J Child Psychol Psychiatry 1997; 38: 633-44.

56. Safer DJ, Allen RD. Hyperactive children: diagnosis and management. Baltimore: University Park Press; 1976

57. Fletcher JM, Shaywitz SE, Shaywitz BA. Comorbidity of learning and attention disorders. Separate but equal. Pediatr Clin North Am 1999; 46: 885-97.

58. Shaywitz BA, Shaywitz SE, Fletcher JM. Learning disabilities and attention disorders, in principles of pediatric neurology. St Louis: Mosby; 2000.

59. Klorman R, Hazel-Fernandez LA, Shaywitz SE, Fletcher JM, Marchione KE, Holahan JM, Stuebing KK, Shaywitz BA. Executive functioning deficits in attention deficit hyperactivity disorder are independent of oppositional defiant or reading disorder. J Am Acad Child Adolesc Psychiatry 1999; 38: 1148-55.

60. Lerner JW. Educational interventions in learning disabilities. J Am Acad Child Adolesc Psychiatry 1989; 28: 32631 .

61. Biederman J, Wilens T, Mick E, Faraone SV, Weber W, Curtis S, Thornell A, Pfister K, Jetton JG, Soriano J. Is ADHD a risk factor for psychoactive substance use disorders? Findings from a four year prospective follow up study. J Am Acad Child Adolesc Psychiatry 1997; 36: 21-9.

62. Shaywitz SE. Dyslexia. Sci Am 1996; 275: 98-104

63. Rourke BP. Nonverbal learning disabilities: the syndrome and the model. New York: Guillford; 1989.

64. Halperin JM, Gittelman R, Klein DF, Rudel RG. Reading disabled hyperactive children: a distinct subgroup of attention deficit disorder with hyperactivity. J Abnorm Child Psychol 1984; 12: 1-14

65. Cunningham CE, Barkley RA. The interactions of normal and hyperactive children with their mothers in free play and structured tasks. Child Dev 1979; 50: 217-24.

66. Routh DK, Schroeder C. Standardized playroom measures as indices of hyperactivity. J Abnorm Child Psychol 1976; 4:199-207.

67. Johnston D, Crawford J, Short H, Smyth TR, Moller J. Poor co-ordination in 5 years olds: a screening test for use in schools. Aust Pediatr J 1987; 23: 157-61.

68. Keller H, Ayub B, Saigal S, Bar-Or O. Neuromotor ability in five to sevenyears old children with very low to extremely low birthweight. Dev Med Child Neurol 1998; 40: 661-6.

68. World Health Organization. International statistical classification of diseases and related health problems. 10th rev. Geneve; 2003.

69. Samango-Sprouse C, Rogol A. The Hidden disability and a prototype for an infantile presentation of developmental dyspraxia (IDD). InfantYoung Child 2002; 15: 11-8.

70. Dewey D. What is developmental dyspraxia? Brain Cogn 1995; $29:$ 254-74.

71. Barowsky EI. Clumsy child. Pediatr Rev 1989; 10: 247-53.

72. Wann JP. Trends in the refinement and optimization of fine motor trajectories: observations from an analysis of the handwriting of primary school children. J Motor Behav 1987; 19: 13-37.
73. Jackson GM, Jackson SR, Husain M, Harvey M, Kramer T, Dow $\mathrm{L}$. The coordination of bimanual prehension movements in a centrally deafferented patient. Brain 2000; 123 (Pt 2): 380-93.

74. Serrien DJ, Nirkko AC, Lovblad KO, Wiesendanger M. Damage to the parietal lobe impairs bimanual coordination. Neuroreport 2001; 12: 2721-4.

75. Fernagut PO, Chalon S, Diguet E, Guilloteau D, Tison F, Jabber M. Motor behavior deficits and their histophathological and functional correlates in the nigrostriatal system of dopamine transporters knockout mice. Neuroscience 2001; 116: 1123-30.

76. Bairstow PJ, Laszlo J. Kinaesthetic sensitivity to passive movements and its relationship to motor development and motor control. Dev Med Child Neurol 1981; 23: 606-17.

77. Hoare D, Larkin D. Kinaesthetic abilities in clumsy children dev Med Child Neurol 1991; 33: 671-78.

78. Walton J, Ellis E, Court S. Developmental apraxia and agnosia. Brain 1965; 85: 603-12.

79. Gillberg C, Carlstrom G, Rasmussen P : Hyperkinetic disorders in seven year old children with perceptual, motor and attentional déficits. J Child Psychol Psychiatr Allied Disc 1983; 24: 233-46.

80. Gillberg C, Carlstrom G, Rasmussen P, Waldenstrom E. Perceptual, motor and attentional deficits in seven year old children neurological screening aspects. Acta Paediatr Scand 1983; 72: 119-24.

81. Connoly K, Stratton P. Developmental changes in associated movements. Dev Med Child Neurol 1968; 10: 49-56.

82. Denckla MB. Revised neurological examination for subtle signs. Psychopharmacol Bull 1985; 21: 773-800.

83. Henderson SE, Sudge D. Movement assessment battery for children. Kent: The Psychological Corporation; 1992

84. Towen BCL. Examination of the child with minor neurological dysfunction. London: SIMP with Heinneman Medical; 1979,

85. Willoughby C, Polatajko H. Motor problems in children with developmental coordination disorder: review of the literature. Am J Occup Ther 1993; 49: 787-94.

86. Laszlo JI, Sainsbury K. Perceptual-motor development and prevention of clumsiness. Psychol Res 1993; 55: 167-74.

87. Losse A, Henderson S, Elliman D, Hall D, Knight E, Jonsgmans M. Clumsiness in children: do they grow out of it? A 10 years follow up study. Dev Med Child Neurol 1991; 33: 32-9.

88. Gillberg IC, Gillberg C. Children with preschool minor neurodevelopmental disorders IV: Behaviour and school achievement at age 13. Dev Med Child Neurol 1989; 31: 313.

89. Henderson L, Rose P, Henderson SE. Reaction time and movement time in children with developmental coordination disorder. J Child Psychol Psychiatry Allied Disc 1992; 33: 895-905.

90. Schoemaker MM, Hijlkema MG, Kalverboer AF. Phisiotherapy for clumsy children: an evaluation study. Dev Med Child Neurol 1994; 36: 143-78.

91. Van Galen GP, Portier SJ, Smits-Engelsman BCM, Schoemaker LRB. Neuromotor noise and poor handwriting in children. Acta Psychol 1993; 82: 161-78. 
92. Smits-Engelsman BC, Niemeijer AS, van Galen GP. Fine motor deficiencies in children diagnosed as DCD based on poor grapho-motor ability. Hum Mov Sci 2001; 20: 161-82.

93. Macnab JJ, Miller LT, Polatajko HJ. The search for subtypes od DCD: is cluster analysis the answer? Hum Mov Sci $2001 ; 20: 49-72$.

94. Blondis TA. Motor disorders and attention déficit/hyperactivity disorder. Pediatr Clin North Am 1999; 5: 899-913.

95. Sandler AD, Watson T, Footo M, Levine MD, Coleman WL, Hooper SR. Neurodevelopmental study of writing disorders in middle childhood. J Dev Behav Pediatr 1992; 13: 16-23.

96. Tervo RC, Azuma S, Fogas B, Fiechtner H. Children with ADHD and motor dysfunction compared with children with ADHD only. Dev Med Child Neurol 2002; 44: 383-90.

97. Raynor AJ Strength, Power and co-activation in children with developmental coordination disorder. Dev Med Child Neurol 2001; 43: 676-84.

98. Pereira HS, Eliasson AC, Forssberg H. Detrimental neural control of precision grip lifts in children with ADHD. Dev Med Child Neurol 2000; 42: 545-53.

99. Pereira HS, Landgren M, Gillberg C, Forssberg H. Parametric control of fingertip forces during precision grip lifts in children with DCD (Developmental Coordination Disorder) and DAMP (Deficits in Attention Motor Control and Perception). Neuropsychologia 2001; 39: 55-68.
100.Eliasson A, Rösblad B, Forssberg H. Disturbances in programming goal direct arm movements in children with ADHD. Dev Med Child Neurol 2004; 46: 19-27.

101. Snow JH, Blondis TA, Accardo PJ, Cunningham KJ. Longitudinal assessment of motor control and sensory skills in academically disabled and control children. Arch Clin Neuropsychol 1993; 8: 55-68.

102.Rasmussen P, Gillberg C. Natural outcome of ADHD with developmental coordination disorder at age 22 years: a controlled, longitudinal, community based study. J Am Acad Child Adolesc Psychiatry 2000; 39: 1424-31.

103.Hellgren L, Gillberg C, Gillberg IC. Children with deficits in attention motor control and perception almost grown up: general health at 16 years. Dev Med Child Neurol 1993; 35 : 881-892.

104.Poltajko HJ, Macnab JJ, Anstett B Malloy-Miller T, Murphy K, Noh S. A clinical trial of the process-oriented treatment approach for children with developmental coordination disorder. Dev Med Child Neurol 1995; 81: 124319.

105.Peeples EE, Searls D, Wellinghan-Jones P. Attention deficit hyperactivity disorder: a longitudinal case study of handwriting characteristics. Percep Motor Skills 1995; 81 : 1243-52.

106.Lerer RJ, Lerer M. The effects of methylphenidate on the soft neurological signs of hyperactive children. Pediatrics 1976; 57: 521-5.

Recebido em 24 de janeiro de 2005

Versão final apresentada em 20 de setembro de 2005

Aprovado em 10 novembro de 2005 